\title{
Application of Trophoblast in Noninvasive Prenatal Testing
}

\author{
Lihua $\mathrm{Wu}^{1,2}$, Ruiyu $\mathrm{Li}^{2}$, Qing $\mathrm{Ye}^{2}$ and Shaopeng Huang ${ }^{2 *}$ \\ ${ }^{1}$ Department of Otolaryngology, Shengli Clinical Medical College of Fujian Medical University, Fujian Provincial Hospital, P.R. \\ China
}

${ }^{2}$ Department of Otolaryngology, Nanfang Hospital, Southern Medical University, Guangzhou, Guangdong, P.R. China

*Corresponding author: Shaopeng Huang, Department of Otolaryngology, Nanfang Hospital, Southern Medical University, Guangzhou, Guangdong, P.R. China

ARTICLE INFO

Received: May 25, 2020

Published: 幽 June 10, 2020

Citation: Lihua Wu, Ruiyu Li, Qing Ye, Shaopeng Huang. Application of Trophoblast in Noninvasive Prenatal Testing. Biomed J Sci \& Tech Res 28(2)-2020. BJSTR. MS.ID.004615.

\section{ABSTRACT}

The application of trophoblast cells in maternal peripheral blood or cervical exfoliated cells for prenatal diagnosis began in the 1980s and 1990s. However, due to the limitations of molecular biology and bioengineering techniques at the time, this technique has only preliminary research on fetal gender, blood type prediction and chromosome analysis and so on. In recent years, with the development of isolation and capture technology of rare cells and biological detection technology, the research of trophoblast in NIPT have been promoted. This article briefly reviews the characteristics of trophoblast cell sorting and its application in NIPT.

\section{Characteristics and Collection Methods of Tropho- blast Cells}

\section{Classification and Characteristics of Trophoblast Cells}

After multiple divisions, the oosperm forms a morula composed of about 32 cells, then the cells begin to differentiate and form blastocyst, The blastocyst wall is trophoblast and the inner layer is inner cell mass. The cells of the inner cell mass are embryonic stem cells, which have totipotency and will develop into fetuses, The trophoblast cells that form the blastocyst wall will develop into the membrane and placenta. Traditional chorionic biopsy is to obtain placental cells dominated by trophoblast cells through puncture for chromosome analysis and gene diagnosis.

The blastocyst is implanted into the endometrium in early pregnancy. After that, trophoblast cells gradually differentiated into two layers. The inner layer which is in contact with the stroma, formerly knownas "langhans cell", now is known as "cytotrophoblast". The outer layer is in contact with the decidua, formerly known as "syncytial cells", now called "syncytiotrophoblast". As trophoblast cells are composed of heterogeneous cells, there are three types depending on the morphological differences: Cytotrophoblast (CT),
Syncytiotrophoblast (ST), and Intermediate Trophoblast (IT). (1) Cytotrophoblast (CT) is monocyte cubic cell, located in the inner layer of villi. (2) Syncytiotrophoblast (ST) is a polynuclear cell that secretes sex hormone and glycoprotein hormones, located in the outer layer of villi. Syncytiotrophoblast is the most differentiated type of trophoblast due to the lack of mitosis. (3) Intermediate trophoblasts (it) are mostly composed of mononuclear cells, which are larger than cytotrophoblasts, but multinuclear cells can also be seen. Intermediate trophoblasts have certain characteristics in common with cytotrophoblasts and syncytiotrophoblasts. However, it is significantly different from cytotrophoblast and syncytiotrophoblast in the characteristics of light microscope, ultrastructure, biochemistry and function. The trophoblasts of villi are mainly cytotrophoblasts and syncytiotrophoblasts. Intermediate trophoblasts are a major component of the extravillous trophoblast. According to the different morphology and surface antibody of the three types of trophoblast cells, it has been confirmed that they exist in the maternal peripheral blood and cervical mucus in the early pregnancy [1-3]. 


\section{Trophoblast Cell Collection Method}

Collecting Trophoblast Cells from Maternal Peripheral Blood: As early as 1893, Schmorl [4] discovered trophoblast cells in the lungs of corpse pregnant women with eclampsia. According to the literature, all three types of trophoblast cells can be found in the peripheral blood of the mother, mainly the trophoblast cells outside the villi5. In 1997, loke, YW et al. [1] reported that the trophoblast cells existed in the maternal blood circulation by the extracellular trophoblast cell-specific antibody HLA-G and FISH detection. The mechanism is speculated as follows; Extravillous trophoblast cells have powerful invasive migration functions. At 8 and 12 weeks of gestation, it can invade the spiral arteries in the endometrium and myometrium, initiate the vascular remodeling and lead to changes in the shape and function of spiral arteries, such as vasodilation, reduction of vascular muscular layer, reduction of resistance, etc [5-7]. These changes increase blood flow from the uterus to the placenta by 40 times to ensure adequate blood perfusion of the placenta [8]. In conclusion, extravillous trophoblasts can invade the spiral artery and appear in the maternal peripheral blood. In addition, some scholars also found these two types of cells in maternal peripheral blood by using cytotrophoblast specific antigen H315 and syncytiotrophoblast specific antigen ft1-41.13. Mouawia et al. [9] reported that more than half of trophoblast cells in maternal peripheral blood were larger than 15 microns in diameter. Currently, the most ideal specific antibodies for placental trophoblast cells are hla-g, beta-hcg and CK [10-12].

It has been reported that trophoblast cells can be collected from maternal peripheral blood at $5 \sim 12$ weeks of gestation and 20 weeks at last $[9,13]$, which is consistent with the time of trophoblast invasion and extension into the spiral artery during the establishment of the maternal-fetal cycle. Depending on the different screening and isolation methods, the number of trophoblast cells captured is different. There are 1-6 trophoblast cells per ml of peripheral blood sample [14,15]. The proportion of nucleated cells in maternal peripheral blood is 1/106 107 [16].

Collection of Trophoblast from Maternal Cervical Exfoliated Cell: In 1971, Shettle [17] first reported the presence of trophoblast cells in cervical mucus of pregnant women, and successfully identified the sex of 10 fetuses by using Y chromosome fluorescence staining. The mechanism which trophoblasts reach the uterine cavity and cervical canal is unclear. It is speculated from the occurrence of villi that the time to collect the trophoblast cells through the cervix is from the invasive growth of villi to the end of uterine cavity disappearance and closure of the internal cervical os [18].

The methods of obtaining cervical exfoliated cells in early pregnancy include: Mucus suction, cell brush or cotton swab, cervix lavage ,intrauterine lavage, etc. Different methods have different success rates [11,19-27] (Table 1). When sampling with cotton swab, It is easy for cells to be involved in cotton fiber and not easy to fall off from cotton swab, which leads to the disadvantage of sampling. At present, this method is basically not adopted. The positive rate and the number of trophoblast cells collected by lavage and suction are slightly higher [12], but there is a risk of uterine infection caused by stimulation of blastocyst, which may cause miscarriage or poor pregnancy. Retrieval of trophoblast cells from the endometrial canal using a cytobrush can be considered minimally invasive. The office procedure is essentially a Pap smear, and can be performed successfully between 5 and 20 weeks GA [12]. Pap smears are recommended during pregnancy, and several studies that surveyed approximately 1900 pregnant women found the cytobrush procedure to be safe and associated with no serious adverse outcomes $[26,28]$. Although cell brush sampling is the safest method, it is most susceptible to the influence of maternal cells and residual sperm and its genetic material, which leads to a wide range of success rate of target cells.

Table 1: Comparison of different methods of transcervical cell sample collection.

\begin{tabular}{|c|c|c|c|}
\hline Method & Description & $\begin{array}{c}\text { Percentage of } \\
\text { transcervical cells } \\
\text { sample with fetal cells }\end{array}$ & References \\
\hline \multirow[t]{2}{*}{$\begin{array}{l}\text { Transcervical smears with } \\
\text { cytobrush }\end{array}$} & $\begin{array}{l}\text { Application of the cytobrush } 2 \text { to } 3 \mathrm{~cm} \\
\text { into the endocervix and } 3600 \text { rotation } \\
\text { while the brush is withdrawn to obtain } \\
\text { cervical mucus. }\end{array}$ & $\begin{array}{c}96.70 \% \\
23.90 \% \\
95 \%\end{array}$ & $\begin{array}{l}20 \\
19 \\
11\end{array}$ \\
\hline & $\begin{array}{l}\text { the cytobrush was not inserted into the endocervical canal } \\
\text { but rather rotated at the external os }\end{array}$ & $100 \%$ & 26 \\
\hline $\begin{array}{l}\text { Aspiration with Pipelle De Cornier } \\
\text { and Aspiracath }\end{array}$ & $\begin{array}{l}\text { Introduction of the Pipelle or Aspiracath into the cervix to } \\
\text { aspirate cervical canal mucus before elective termination } \\
\text { of pregnancy. }\end{array}$ & $50 \%$ & 22 \\
\hline \multirow{5}{*}{ Endocervical canal lavage } & Application of a flexible plastic catheter & \multirow{5}{*}{$\begin{array}{c}83.30 \% \\
50 \% \\
59.20 \%\end{array}$} & \multirow{3}{*}{$\begin{array}{l}23 \\
21\end{array}$} \\
\hline & connected to a 10 -mL syringe into the & & \\
\hline & endocervix which is flushed and & & \\
\hline & immediately aspirated back to obtain & & \multirow{2}{*}{25} \\
\hline & cellular content. & & \\
\hline
\end{tabular}




\begin{tabular}{|c|c|c|c|}
\hline $\begin{array}{l}\text { Intrauterine lavage with or without } \\
\text { ultrasound guidance }\end{array}$ & $\begin{array}{l}\text { Use of a thin flexible plastic catheter } \\
\text { connected to a } 10 \text {-mL syringe, which } \\
\text { is introduced via the internal cervical } \\
\text { os with or without transabdominal } \\
\text { ultrasound guidance before } 5 \mathrm{~mL} \text { of } \\
\text { saline is instilled and aspirated back } \\
\text { under gentle suction. }\end{array}$ & $\begin{array}{c}84.80 \% \\
82 \% \\
92.3 \% \quad \text { (with ultrasound } \\
\text { guidance) }\end{array}$ & $\begin{array}{l}27 \\
19 \\
23\end{array}$ \\
\hline
\end{tabular}

The earliest time of collecting trophoblast cells through the cervix by different methods was $5 \sim 7$ weeks of pregnancy, and the latest time was $13 \sim 20$ weeks of pregnancy [12,18,29]. It is reported that the number of trophoblast cells collected from the cervix at 9 weeks of gestation decreased, which is statistically significant compared with that collected at 6, 7 and 8 weeks of gestation [30]. In addition, the number of trophoblast cells collected through the cervix was not affected by maternal age or obesity [12]. The time of collecting trophoblastic cells from the cervix was basically the same as that from maternal peripheral blood. In normal pregnant cervical exfoliated cells, the proportion of trophoblast cells collected by cell brush is about 1:200011, which is far more than the number of trophoblast cells collected from peripheral blood [31].

To sum up, on the one hand, trophoblast cells are derivatives of mitosis of zygote, which can accurately reflect fetal genetic characteristics to some extent, on the other hand, the existence of placental trophoblast cells in maternal peripheral blood and cervical exfoliated cells has been confirmed by many scholars, and the cell size and morphological characteristics are obvious. It is easy to identify and isolate trophoblast cells with ideal specific antibodies, and the integrity of DNA of these cells is verified by terminal transferase labeling, which contains all the genetic material of the fetus and is not affected by multiple pregnancy [32]. Therefore, it is feasible to collect placental trophoblast cells from maternal peripheral blood or cervical mucus for noninvasive prenatal test.

\section{Sorting of Trophoblast Cells}

\section{Separation of Trophoblast Cells:}

The separation of trophoblast cells is an important part of minimally invasive prenatal diagnosis based on cervical exfoliated trophoblasts. The purity and concentration of the separated cells directly affect the accuracy of the diagnosis results.

Density Gradient Centrifugation: Density gradient centrifugation is often used as the first step in the enrichment of maternal peripheral blood trophoblast cells.

Micromanipulatio: Micromanipulation is simple in operation. Under the microscope, cells with trophoblast and plush characteristics in the specimen are directly drawn out through glass tube, but the method relies on manual operation and has a low degree of automation. This method is often used in conjunction with other cell sorting methods.
Immunomagnetic Separation and Flow Cytometry: Immunomagnetic separation and flow cytometry, based on the specific binding of trophoblast cell-expressing antigen and its antibody to separate target cells, At present, HLA-G, $\beta$ - hCG and CK are the most widely used placental trophoblast specific antibodies [10-12]. G233 is the specific antibody of Human Leukocyte Antigen G (HLA-G) expressed by extracellular trophoblast cells and intermediate trophoblast cells. The magnetic beads coupled with the antibody bind to the target cells, and under the action of the external magnetic field, the complexes stay in the magnetic field to separate the cells, and the purity of the isolated cells is up to $90 \%$ $100 \%, 500 \sim 1500$ trophoblastic cells can be obtained from each cervical mucus specimen, the isolated cells have the phenotype and intact nuclear DNA (> 95\%) of trophoblastic cells [33]. Flow cytometry uses HLA-G fluorescent antibodies to bind to trophoblast cells to sort out high-purity trophoblast cells. This technique is also suitable for cytoplasmic antigens. The target cells isolated by these two methods are high in purity, but a certain amount of target cells are lost due to factors such as high specificity of antibodies and antigen destruction caused by pretreatment of specimens.

Laser Capture Microdissection (LCM): Laser Capture microdissection (LCM), a cell purification technique based on cell shape, size, and cytopathology, can capture and microcut specific cells under the microscope. Mouawia et al. [9] reported that more than half of the trophoblast cells in the maternal peripheral blood were larger than $15 \mu \mathrm{m}$ in diameter, and the trophoblast cells in maternal peripheral blood were successfully sorted and captured by LCM. Combined with immunohistochemistry and in situ hybridization techniques, the efficiency of cell purification by this technique is greatly improved. but attention should also be paid to the damage of the nucleus and cytoplasm caused by the cutting precision.

Differential Adhesion Method: Differential adhesion method uses the different adherent speed of trophoblast cells and maternal contaminated cells to remove maternal suspended cells. In Yuan Jing et al's research [34], the success rate of trophoblast cells in differential adherent culture was $95 \%$, and the gender of $90 \%$ of the fetuses was successfully identified. The culture success rate of the cells in the direct culture control group was $96 \%$, but only $38 \%$ of fetuses' gender was correctly identified.

Microfluidic Chip Technology: Microfluidic chip technology integrates basic operations such as sample preparation, reaction, 
separation and detection into a micron scale chip. One example is Abnova's CytoQuest ${ }^{\mathrm{TM}}$ CR microfluidic system. Microfluidic chip micron-sized channel is suitable for single cell introduction, manipulation, reaction, separation and detection, and is more suitable for samples of peripheral blood to separate and capture trophoblast cells.

\section{Identification of Trophoblast Cells}

Fetal origin identification of isolated cells is an essential step for prenatal diagnosis with trophoblast cells, and it is also the key to achieve accurate prenatal diagnosis. The isolated cells were confirmed as fetal cells by cell morphology, immunophenotype and genetic analysis, so as to further used for prenatal diagnosis.

a) The morphological method is to observe the Hematoxylin-Eosin staining (HE) results of the cervical smear under the microscope, and identify the trophoblast cells from many contaminated maternal cells. Cervical trophoblast cells include cytotrophoblast cells, intermediate trophoblast cells and syncytiotrophoblast cells, among which syncytiotrophoblast cells have unique morphology, variable cell size and shape, The nucleus is small and deeply stained, with different numbers and uniform size, the cytoplasm is weakly eosinophilic and easy to distinguish, but the morphological identification method is subjective, and the suspected cells need to be determined by immunohistochemistry or genetic analysis.

b) Immunohistochemical staining using specific antigen epitopes expressed by trophoblast cells is a common method for cell identification. Commonly used identification antibodies include G233, anti-Cytokeratin 7 (ck-7) antibody, Anti-Human Chorionic Gonadotropin (hCG) antibody, NDOG5, NDOG1 and FT141. 1 etc. G233 and NDOG5 specifically bind to cytotrophoblast cells [1,18]; NDOG1 recognizes syncytiotrophoblast cells [30]; FT141.1 recognizes all trophoblast cells [22]. $\beta$-hCG is a specific protein secreted by trophoblast cells, which has been used to identify the purity of isolated cells [35]. Anti-CK-7 antibodies are low in specificity, and the false negative result caused by the specific antibody can be counteracted by the combined staining of anti-ck-7 antibody and high specific antibody [11]. Insufficient antibody specificity or sensitivity of antibodies and antigen destruction caused by pretreatment of specimens will reduce the efficiency of cell identification by immunohistochemistry. It is necessary to explore combinations of antibodies with strong specificity and high sensitivity, or to combine with molecular biological analysis to offset the lack of methodology.

Genetic Information Analysis Method: Currently, the most widely used genetic information analysis methods are Fluorescence In Situ Hybridization (FISH) and quantitative fluorescence PCR (QF-PCR) etc., which identify the fetal origin by identifying the genetic information of fetal cells different from the mother. FISH obtains the information of chromosome or gene status in the nucleus by hybridizing a fluorescein-labeled DNA probe to a DNA target sequence in the sample nucleus. For example, DYZ1 satellite III on the Y chromosome and DXZ1 satellite alpha DNA on the X chromosome, can be used to distinguish the fetal and maternal cells by evaluating the positive nucleus, and identify the contaminated sperm [10]. Because FISH directly labels specific genes or groups of genes with fluorescent dyes, it is possible to visualize the number of copies of genes or chromosomes within individual cells. A wide variety of FISH probes are available that can be applied in the detection of aneuploidies on freshly fixed cells or fixed tissues stored in paraffin blocks. Based on DNA polymorphism, fluorescent quantitative PCR introduces highly polymorphic markers such as Short Tandem Repeat (STR), which are distinguished from the STR spectra of maternal cells by the heterozygosity of the individual. Due to the introduction of highly polymorphic markers such as pentanucleotide and hypoxanthine-guanine phosphateribosyltransferase STR in the long-arm pseudo-chromosome region of the $\mathrm{X}$ and $\mathrm{Y}$ chromosomes makes the detection unaffected by the gender of the specimen fetuses, In addition, aneuploidy analysis can be performed by using the fluorescence peak ratio of the given STR site [30]. The use of QF-PCR for prenatal diagnosis eliminates the need to culture and expand fetal cells and allows rapid diagnosis of some selected chromosomal anomalies $(13,18,21$, and $X$ and Y). Compared with standard karyotyping, FISH and QF-PCR can be exquisitely sensitive for the analysis of TCC and IUL samples.

\section{Application of Trophoblast Cells in Prenatal Diag- nosis}

\section{Early Application of Trophoblast Cells for Noninvasive Prenatal Testing}

In the early stage, due to the lack of rare cell separation technology and the extremely low proportion of fetal cells in maternal peripheral blood, it is mainly based on the cytogenetic technology mainly including PCR and fish to identify trophoblast cells, and to engage in the research of gender prediction, chromosomal aneuploid disease prediction and blood type prediction

Peripheral blood trophoblast cells: As early as 1971, Nature reported that Shettles used cervical mucus smear in early pregnancy for fluorescent staining, and found the existence of Y-body positive cells [17]. After the rise of PCR technology, in 1992, many scholars extracted DNA from the cervical exfoliated cells of pregnant women with male fetuses, designed Y-chromosome specific fragment primers, and detected the amplified of Y-chromosome specific fragment after PCR amplification and sequencing [36]. In 1995, Kingdom et al. [37] used Short Tandem Repeat (STR) as a genetic marker and fluorescence quantitative PCR technology, In 11 cases of cervical ectomized cells collected frome pregnant women by intrauterine lavage, 5 cases detected paternal STR. 8 cases of cervical mucus were collected by cell brushing, and 1 case detected paternal STR. With the advent of fish technology, the proportion of fetal cells in cervical exfoliated cells can be predicted. In the early stage, due 
to the lack of rare cell separation technology, the proportion of fetal cells in maternal peripheral blood was extremely low, Only the proportion of fetal trophoblast cells could be roughly estimated and the fetal chromosome aneuploidy disease could be predict by manual counting of cervical smear samples under microscope.

\section{Cervical Trophoblast Cells}

The accuracy of early use of trophoblast cells for gender prediction is different due to different methods of collection and analysis. Morri and Williamson used the cells collected by intrauterine lavage to amplify Y-DNA by PCR [38], only 4 of 13 cases of pregnant women with male fetuses amplified Y-DNA. In 2001, Mosher [20] collected cervical mucus from 30 pregnant women (610 weeks of gestation) who wanted to undergo induced abortion, and used two-color fish technology to detect X and Y chromosomes to predict the gender of the fetus. At the same time, the results of chromosome analysis in abortion tissue culture were used as gold standard control. The results showed that among the 13 males, 11 cases detected Y chromosome, Y chromosome was not detected in maternal cervical cells in 1 case, 1 case of trophoblast cell showed 46, XY /47,XXY (25\%) chimerism by FISH, while karyotype analysis showed 46,XY. In 2010, Sifakis [39] collected 5 cases of cervical mucus smears (about 200,000 cells/slide) of 5 pregnant women (11-13 weeks of gestation) whose fetus was confirmed as 21 trisomy by chorionic biopsy. After FISH test, 21-trisomy cells were found in all the 5 pregnant women's cervical exfoliated cells, with a count of 1-27 cells/slide. In 1995, Adinolfi et al. [18] used PCR to detect cervical mucus samples of 12 pregnant women with $\mathrm{RhD}$ negative blood group after $\mathrm{RhD}$ gene amplification, and the prediction accuracy of fetal Rh blood group was less than $50 \%$.

\section{Progress in NIPT of Trophoblast Cells with the Development of Isolation and Capture of Rare Cells}

The development of isolation and capture technology of rare cells and molecular biology technology has provided new ideas and new models for the application of trophoblast cells in NIPT. Brechot [32] used laser capture microdissection technology to separate trophoblast cells from peripheral blood of pregnant mother (4-12 weeks gestation). After genome-wide amplification, STR classification was used to identify whether the cells were fetal origin. NIPT was carried out in 63 pregnant women with progressive spinal muscular atrophy (SMA, 31 cases) or cystic fibrosis (CF, 32 cases). 14 suffering fetuses were detected, and confirmed by chorionic biopsy results. The sensitivity of diagnosis was $95 \%$ (confidence interval 76.8-100\%) and the specificity was 95\% (confidence interval 92.7-100\%).

Breman A.M [32] used microfluidic technology to sort CK+ and CD45-trophoblast cells in maternal peripheral blood and captured 0.36 trophoblast cells per milliliter of peripheral blood on average. After whole-genome amplification, the fetal origin was determined by STR classification. After whole-genome amplification of isolated trophoblast cells, chromosome aneuploidy (13/18/21/X/Y) and $2.7 \mathrm{Mb}$ microdeletions were detected by arrayCGH and Next Generation Sequencing for the first time.

Jain, C. V 10 collected cervical mucus from 20 pregnant women (5-20 weeks gestation) that before induced abortion, sorted trophoblast cells by MACS technique, and developed a "nuclear separation method" to remove most of the extracellular maternal DNA fragments. The Next Generation Sequencing was conducted (detection sites include 94 SNPs and 59 STRs distributed on all chromosomes), and Next Generation Sequencing results of aborted fetal cells were used as gold standard controls to calculate the proportion of maternal contamination. The results showed that the purity of fetal DNA was $92.2 \% \pm 6.5 \%$ in the early pregnancy (the earliest is 5 weeks gestation), and the correct rate of fetal genotype was $100 \%$. However, for the current NIPT based on fetal free nucleic acid, the proportion of fetal DNA in maternal peripheral blood free DNA is only $4 \%$ to $10 \%$ at the 10 th week of pregnancy. Their results suggested that cervical trophoblast cells can provide an earlier and more accurate prenatal genetic screening method.

Sifakis S et al. [39] used cell brush to obtain cervical mucus from 11-13 weeks pregnant women, and accurately diagnosed 5 fetuses with Down's syndrome through FISH analysis. The largest number of triploid cells in a single sample was up to 27. Biron-shantal et al. [40] strengthened the understanding of cervical trophoblast cells in early pregnancy. By comparing the fish hybridization results of cervical specimens and placental tissues in early pregnancy, it was found that in normal pregnancy, there were polyploid cells caused by intranuclear replication of trophoblast cells, but most of them were tetraploid, which would not lead to false-positive triploid.

Pfeifer et al. [26] successfully screened 3 cystic fibrosis and 3 spinal muscular atrophy fetuses from 21 cervical specimens of 8-12 gestational weeks pregnant women by single cell gene expression profiling. In addition, the researchers also used the number of cervical trophoblast cells or the difference in proteins expressed by the cells to predict adverse pregnancy outcomes [11].

\section{Conclusion}

Compared with NIPT which is based on fetal free nucleic acid, NIPT based on placental trophoblast cells can provide complete genetic information of fetuses and open a new direction for the development of NIPT. Placental trophoblast cells have the advantages of being available in the early stage of pregnancy ( 5 weeks), having obvious morphological characteristics, having ideal specific antibodies and being easy to identify and separate. Moreover, the number of trophoblast cells obtained by cervical collection is more than that obtained by peripheral blood collection, and it is easy to obtain enough fetal genetic material. With the continuous development of molecular biology, placental trophoblast cells will cover more studies on birth defects in NIPT in the future. 


\section{References}

1. Loke YW, King A, Burrows T, Gardner L, Bowen M, et al. (1997) Evaluation of trophoblast HLA-G antigen with a specific monoclonal antibody. Tissue Antigens 50: 135-146.

2. van Wijk IJ, Griffioen S, Tjoa ML, Mulders MA, van Vugt JM, et al. (2001) HLA-G expression in trophoblast cells circulating in maternal peripheral blood during early pregnancy. Am J Obstet Gynecol 184: 991-997.

3. Johansen M, Redman CW, Wilkins T, Sargent IL (1999) Trophoblast deportation in human pregnancy--its relevance for pre-eclampsia. Placenta 20: 531-539.

4. Lapaire O, Holzgreve W, Oosterwijk JC, Brinkhaus R, Bianchi DW (2007) Georg Schmorl on trophoblasts in the maternal circulation. Placenta 28 $1-5$.

5. Hatt L, Marie Brinch, Ripudaman Singh, Kristine Møller, Rune Hoff Lauridsen, et al. (2014) Characterization of fetal cells from the maternal circulation by microarray gene expression analysis--could the extravillous trophoblasts be a target for future cell-based non-invasive prenatal diagnosis? Fetal Diagn Ther 35: 218-227.

6. Harris LK, Rosemary J Keogh, Mark Wareing, Philip N Baker, Judith E Cartwright, et al. (2006) Invasive trophoblasts stimulate vascular smooth muscle cell apoptosis by a fas ligand-dependent mechanism. Am J Pathol 169: 1863-1874.

7. Ashton SV, Guy St J Whitley, Philip R Dash, Mark Wareing, Ian P Crocker, et al. (2005) Uterine spiral artery remodeling involves endothelial apoptosis induced by extravillous trophoblasts through Fas/FasL interactions. Arterioscler Thromb Vasc Biol 25: 102-108.

8. Chang K, Lubo Zhang (2008) Review article: steroid hormones and uterine vascular adaptation to pregnancy. Reprod Sci Thousand Oaks Calif 15: 336-348.

9. Mouawia H, Ali Saker, Jean-Philippe Jais, Alexandra Benachi, Laurence Bussières, et al. (2012) Circulating trophoblastic cells provide genetic diagnosis in 63 fetuses at risk for cystic fibrosis or spinal muscular atrophy. Reprod Biomed Online 25: 508-520.

10. Jain CV, Leena Kadam, Marie van Dijk, Hamid-Reza Kohan-Ghadr, Brian A Kilburn, et al. (2016) Fetal genome profiling at 5 weeks of gestation after noninvasive isolation of trophoblast cells from the endocervical canal. Sci Transl Med 8: 363re4.

11. Imudia AN, Yoko Suzuki, Brian A Kilburn, Frank D Yelian, Michael P Diamond, et al. (2009) Retrieval of trophoblast cells from the cervical canal for prediction of abnormal pregnancy: A pilot study. Hum Reprod Oxf Engl 24: 2086-2092.

12. Fritz R, Hamid Reza Kohan-Ghadr, Alex Sacher, Alan D Bolnick, Brian A Kilburn, et al. (2015) Trophoblast Retrieval and Isolation from the Cervix (TRIC) is unaffected by early gestational age or maternal obesity. Prenat Diagn 35: 1218-1222.

13. Guetta E, Gutstein-Abo L, Barkai G (2005) Trophoblasts isolated from the maternal circulation: in vitro expansion and potential application in non-invasive prenatal diagnosis. J Histochem Cytochem Off J Histochem Soc 53: 337-339.

14. Hatt L, Marie Brinch, Ripudaman Singh, Kristine Møller, Rune Hoff Lauridsen, et al. (2014) A new marker set that identifies fetal cells in maternal circulation with high specificity. Prenat Diagn 34: 1066-1072.

15. Emad A, Drouin R (2014) Evaluation of the impact of density gradient centrifugation on fetal cell loss during enrichment from maternal peripheral blood. Prenat Diagn 34: 878-885.

16. Hahn S, Holzgreve W (2002) Prenatal diagnosis using fetal cells and cellfree fetal DNA in maternal blood: what is currently feasible? Clin Obstet Gynecol 45: 649-656.

17. Shettles LB (1971) Use of the $\mathrm{Y}$ chromosome in prenatal sex determination. Nature 230: 52-53.
18. Adinolfi M, Sherlock J (1997) First trimester prenatal diagnosis using transcervical cells: An evaluation. Hum Reprod Update 3: 383-392.

19. Cioni R, Cecilia Bussani, Benedetta Scarselli, Sandra Bucciantini, Mauro Marchionni, et al. (2005) Comparison of two techniques for transcervical cell sampling performed in the same study population. Prenat Diagn 25: 198-202.

20. Fejgin MD, Diukman R, Cotton Y, Weinstein G, Amiel A (2001) Fetal cells in the uterine cervix: A source for early non-invasive prenatal diagnosis. Prenat Diagn 21: 619-621.

21. Bulmer JN, Riccardo Cioni, Cecilia Bussani, Vincenzo Cirigliano, Francesc Sole, et al. (2003) HLA-G positive trophoblastic cells in transcervical samples and their isolation and analysis by laser microdissection and QF-PCR. Prenat Diagn 23: 34-39.

22. Katz-Jaffe MG, Mantzaris D, Cram DS (2005) DNA identification of fetal cells isolated from cervical mucus: potential for early non-invasive prenatal diagnosis. BJOG 112: 595-600.

23. ErgIn T, Baltaci V, Zeyneloglu HB, Duran EH, ErgenelI MH, et al. (2001) Non-invasive early prenatal diagnosis using fluorescent in situ hybridization on transcervical cells: comparison of two different methods for retrieval. Eur J Obstet Gynecol Reprod Biol 95: 37-41.

24. Fang CN, Kan YY, Hsiao CC (2005) Detection of fetal cells from transcervical mucus plug before first-trimester termination of pregnancy by cytokeratin-7 immunohistochemistry. J Obstet Gynaecol Res 31: 500-507.

25. Bahado-Singh RO, Kliman H, Feng TY, Hobbins J, Copel JA, et al (1995) First-trimester endocervical irrigation: feasibility of obtaining trophoblast cells for prenatal diagnosis. Obstet Gynecol 85: 461-464

26. Pfeifer I, Benachi A, Saker A, Bonnefont JP, Mouawia H, et al. (2016) Cervical trophoblasts for non-invasive single-cell genotyping and prenatal diagnosis. Placenta 37: 56-60.

27. Bussani C, Riccardo Cioni, Alberto Mattei, Massimiliano Fambrini, Mauro Marchionni, et al. (2007) Prenatal diagnosis of common aneuploidies in transcervical samples using quantitative fluorescent-PCR analysis. Mol Diagn Ther 11: 117-121.

28. Holt J, Stiltner L, Jamieson B, Fashner J (2005) Clinical inquiries. Should a nylon brush be used for Pap smears from pregnant women? J Fam Pract 54: 463-464.

29. Imudia AN, Kumar S, Diamond MP, DeCherney AH, Armant DR (2010) Transcervical retrieval of fetal cells in the practice of modern medicine: a review of the current literature and future direction. Fertil Steril 93: 1725-1730.

30. Mantzaris D, Cram DS (2015) Potential of syncytiotrophoblasts isolated from the cervical mucus for early non-invasive prenatal diagnosis: Evidence of a vanishing twin. Clin Chim Acta Int J Clin Chem 438: 309 315.

31. Bischoff FZ, Marquéz-Do DA, Martinez DI, Dang D, Horne C, et al. (2003) Intact fetal cell isolation from maternal blood: improved isolation using a simple whole blood progenitor cell enrichment approach (RosetteSep). Clin Genet 63: 483-489.

32. Breman AM, Jennifer C Chow, Lance U Ren, Elizabeth A Normand, Sadeem Qdaisat, et al. (2016) Evidence for feasibility of fetal trophoblastic cellbased noninvasive prenatal testing. Prenat Diagn 36: 1009-1019.

33. Bolnick JM, Brian A Kilburn, Swati Bajpayee, Nitya Reddy, Roohi Jeelani, et al. (2014) Trophoblast retrieval and isolation from the cervix (TRIC) for noninvasive prenatal screening at 5 to 20 weeks of gestation. Fertil Steril 102: 135-142

34. Yuan Jing, Fu Juanjuan, Chen Wei, Fang Huiqin, Zong Lin (2014) A preliminary study on the use of exfoliated trophoblasts in the cervix for prenatal diagnosis in early pregnancy. Chin J Prenat Diagn Electron 4: 6-10. 
35. Bolnick JM, Hamid-Reza Kohan-Ghadr, Rani Fritz, Alan D Bolnick, Brian A Kilburn, et al. (2016) Altered biomarkers in trophoblast cells obtained noninvasively prior to clinical manifestation of perinatal disease. Sci Rep 6: 32382 .

36. Zimmerman R, Huch A, Kratzer A, Bär W (1993) Detection of fetal DNA in trans-cervical swabs from first trimester pregnancy by gene amplification: A new route to prenatal diagnosis? Br J Obstet Gynaecol 100: 400 .

37. Kingdom J, Sherlock J, Rodeck C, Adinolfi M (1995) Detection of trophoblast cells in transcervical samples collected by lavage or cytobrush. Obstet Gynecol 86: 283-288.

ISSN: 2574-1241

DOI: $10.26717 /$ BJSTR.2020.28.004615

Shaopeng Huang. Biomed J Sci \& Tech Res

(C) This work is licensed under Creative

Submission Link: https://biomedres.us/submit-manuscript.php
38. Gammill HS, Nelson JL (2010) Naturally acquired microchimerism. Int J Dev Biol 54: 531-543.

39. Sifakis S, Satish Ghatpande, Antti Seppo, Michael W Kilpatrick, Triantaphyllos Tafas, et al. (2010) Prenatal diagnosis of trisomy 21 through detection of trophoblasts in cervical smears. Early Hum Dev 86: 311-313.

40. Biron-Shental T, Moshe D Fejgin, Stavros Sifakis, Meytal Liberman, Aris Antsaklis, et al. (2012) Endoreduplication in cervical trophoblast cells from normal pregnancies. J Matern Fetal Neonatal Med 25: 2625-2628.

$\begin{array}{ll}\text { BIOMEDICAL } & \text { Assets of Publishing with us } \\ \text { RESEARCHES } & \text { - Global archiving of articles } \\ \text { - Immediate, unrestricted online access } & \text { - Rigorous Peer Review Process } \\ & \text { - Authors Retain Copyrights } \\ \end{array}$

